Investigation of the effect of the water to powder ratio on hydraulic cement properties ${ }^{1}$ Koutroulis A, ${ }^{2}$ Batchelor H, ${ }^{1}$ Kuehne SA, ${ }^{1}$ Cooper PR, ${ }^{1}$ Camilleri J

${ }^{1}$ School of Dentistry, Institute of Clinical Sciences, College of Medical and Dental Sciences, University of Birmingham, ${ }^{2}$ School of Pharmacy, Institute of Clinical Sciences, College of Medical and Dental Sciences, University of Birmingham, Birmingham, United Kingdom

Key words:

Tricalcium silicate, rheology, characterisation, water to powder ratio

\author{
Correspondence \\ Josette Camilleri \\ School of Dentistry, \\ Institute of Clinical Sciences, \\ College of Medical and Dental Sciences, \\ University of Birmingham \\ 5 Mill Pool Way \\ Edgbaston B5 7EG \\ Birmingham \\ United Kingdom \\ J.Camilleri@bham.ac.uk
}




\section{Investigation of the effect of the water to powder ratio on hydraulic cement properties}

\section{Abstract}

Objectives. The use of rheological properties to determine the optimal water: powder ratio of tricalcium silicate-based prototype materials incorporating alternative radiopacifiers and fillers. Determination of how the proportion of water incorporated affected the physicochemical behaviour of the materials.

Methods. Endodontic cements replaced with $30 \%$ radio-opacifier, and additions of calcium phosphate and micro-silica were tested. The unmodified cements were mixed with a 0.35 water: powder ratio which served as control. At this water: powder ratio, the paste had an adequate clinical consistency and furthermore these pastes have been well characterized. Assessment of material rheological properties enabled adjustment of the water: powder ratio in each material to provide comparable viscosity values to those of the controls. The flowability, phase analysis and calcium release were measured for both viscosity-matched and the standard 0.35 water: powder ratio blends. The prototype materials with the adjusted water ratio were also characterized by scanning electron microscopy, energydispersive spectroscopy and evaluated for radio-opacity.

Results. The use of the 0.35 water: powder ratio is not appropriate when changing the radiopacifier and incorporating additives. Zirconium oxide did not vary the water: powder ratio but tantalum oxide and calcium tungstate resulted in an increase and decrease in water demand respectively. Using the standard 0.35 ratio when the mixture had a low water demand resulted in higher flowability values and calcium release in solution. Micro-silica and calcium phosphate altered the hydration of the materials. All materials were adequately radiopaque.

Significance. Rheological assessment is an easy reproducible way to determine the 
water: powder ratios of materials with varying amounts of additives and radiopacifiers during development. Modifications to the water: powder ratio affects material properties.

\section{Introduction}

Tricalcium silicate (TCS) -based materials were introduced in dentistry through the use of Portland cement (PC) in mineral trioxide aggregate (MTA) [1]. The initial formulation of MTA, consisting of $80 \%$ PC and $20 \%$ bismuth oxide as radiopacifier [1], has been widely used for endodontic applications such as root-end filling [2], root-repair [3] and vital pulp therapy $[4,5]$. However, several limitations exist in the original formulation, such as the inclusion of trace elements namely lead, chromium and arsenic in the cementitious phase [6-8] as well as the leaching of bismuth [9] which results in tooth discoloration [10].

In an effort to eliminate the leaching of trace elements from Portland cement, pure TCS is now used in several commercially available hydraulic cements $[11,12]$ and various compounds are also included to enhance the cement's physicochemical properties [13-15]. Similarly, inert materials with high molecular weight and which do not leach out have been incorporated as radiopacifiers [16]. Indeed it is important that compounds which serve as radiopacifiers in TCS-based formulations remain stable following cement hydration as a reduction in radio-opacity of the material can occur in the application field over long-term

clinical use. Notably zirconium oxide, tantalum pentoxide and calcium tungstate have been used in commercial materials for this purpose [17-19].

TCS-based materials are mixed with water to obtain a homogeneous mixture. The cement particles hydrate in contact with the water and produce mainly calcium silicate hydrate and calcium hydroxide [9]. ProRoot MTA is mixed with water in a 0.33 powder to liquid ratio [4] as indicated by the manufacturer. A similar ratio is frequently used in studies 
to assess properties of experimental TCS-based materials $[15,20]$. Notably when the water: powder ratio is not sufficient to hydrate the mixture, alterations in the amount of water used are typically undertaken using visual inspection of the mixture by the operator to obtain acceptable consistency and handling efficiency. It has also been shown in experimental materials that addition of calcium phosphate or silica-based compounds, alters the hydration process of the cement [20-22].

Although a lot of work is undertaken to create novel materials based on TCS for clinical use, to date, there is no data in the literature regarding the exact alterations in the water demand in the powder to liquid proportion of hydraulic cements following replacement by different compounds and radiopacifiers in their formulation in order to be adequately hydrated. Notably, it has been reported that modifications in the water amount used in the mixture can have a significant effect in the physicochemical properties of cements $[23,24]$. To the best of our knowledge, an accurate protocol for the determination of the water: powder proportion in hydraulic cements is still lacking.

Rheological properties of TCS-based materials with a potential of use as endodontic

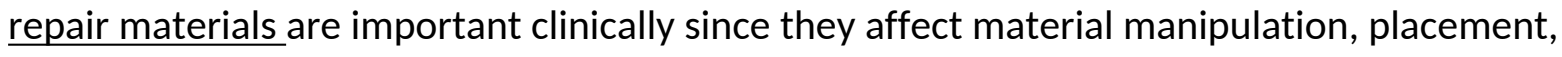
and stability. So far the flowability of hydraulic cements has not been extensively studied [25-27]; however, flow properties of TCS-based endodontic sealers are more routinely reported in the literature $[18,28,29]$. This could be attributed to the lack of a standardised model for flowability tests on hydraulic cements. The aim of this research therefore was to develop a standardized model for the calculation of water: powder ratio for hydraulic calcium silicate materials based on rheological properties and to investigate if the adjustment of the water: powder ratio affects the physicochemical properties of the materials. The null hypothesis was that the various additions will not affect the water 
demand of the hydraulic calcium silicate cements. The other hypothesis studied was that materials mixed at different water: powder ratios will not present alterations in their physicochemical properties.

\section{Materials and Methods}

The materials studied were:

- Tricalcium silicate cement (TCS; Mineral Research Processing, Meyzieu, France)

- TCS and various radiopacifiers replacing the cement by $30 \%$ proportion including zirconium oxide (ZrO; CAS No: 1314-23-4) , tantalum oxide (TaO; CAS No: 1314-61-0), calcium tungstate (CaWO; CAS No: 7790-75-2) and a mixture of zirconium oxide and tantalum oxide in 15:15 wt\% proportion (Sigma Aldrich, Gillingham, UK)

- $\quad$ Radiopacified TCS with 15 wt\% replacement of calcium phosphate monobasic (CaP; Sigma Aldrich, Gillingham, UK, CAS No: 7758-23-8)

- Radiopacified TCS with 10 and 20 wt\% addition of micro-silica (mS10, mS20 respectively; Meyco 610, MBT-FEB, Manchester, UK)

- Portland cement (PC; Blue Circle Portland Cement, Tarmac, Wolverhampton, UK, CAS No: 65997-15-1)

All the testing was performed in triplicate.

\subsection{Rheological assessment}

Portland cement was used in this experimental model system as this has been studied in the construction industry [30] and large quantities were available manufactured from a single batch. Portland cement manufactured to BS EN 197-1: 2000, type CEMI $52.5 \mathrm{~N}$ was mixed with water at a water: powder ratio of 0.35 (PC 0.35$)$. This formulation was regarded as providing the ideal mixture with a clinically acceptable consistency and its hydration has been described thoroughly [11]. This water: powder ratio was used as the 
baseline for all the experiments. The performance of Portland cement mixed with water in the above ratio served as a control for the establishment of the optimum proportion of water in all the other materials with additives or radiopacifiers.

A shear rheometer with adjustable temperature (Discovery HR-1, TA instruments, New Castle, USA) with stainless steel parallel plate geometry and a $40 \mathrm{~mm}$ diameter upper plate with crosshatched surfaces was used. After the mixing procedure using $2.5 \mathrm{~g}$ of each powder with the respective amount of water undertaken upon a glass plate with a metal spatula, an addition of $87.5 \mu \mathrm{L}$ of water per gram was transferred to the initial mixture to ensure reproducible sample loading and homogenous contact surface with the upper plate.Samples were then loaded immediately upon the Peltier plate. The gap between the two plates was set at $700 \mu \mathrm{m}$ and the temperature of the Peltier plate was established at $\underline{20^{\circ} \mathrm{C}}$. The upper plate was initially loaded at $800 \mu \mathrm{m}$, where any potential excess of the material that was squeezed out was removed. Experiments were performed with a 2-minute flow-sweep test, during which the shear rate applied escalated from 1 to $100 \mathrm{~s}^{-1}$. The prototype materials were mixed with different water: powder ratios in order to obtain flowability values comparable with those of the control. Results of the values of viscosity per shear rate from 1 to $20 \mathrm{~s}^{-1}$ were plotted, as at higher shear rates the measurements were not always related to the samples as there was potential for separation of the upper plate from the sample. An evaluation of the tricalcium silicate was also performed and the values were translated to the TCS-based mixtures.

\subsection{Flowability measurement}

Flowability tests of the materials mixed either in a 0.35 or an adjusted water: powder ratio established by the rheometer test were performed. The International Standards for dental root canal sealing materials (ISO 6876:2012) instructions for 
assessment of flowability were followed [31]. More specifically, $0.05 \mathrm{~mL}( \pm 0.005 \mathrm{~mL})$ of each material was transferred to a $40 \mathrm{~mm}$ x $40 \mathrm{~mm}$ glass plate ( $5 \mathrm{~mm}$ thick) with the use of a graduated syringe (BD Plastipak, Wokingham, UK). Two minutes after the start of mixing, a similar glass plate, weighing $20 \mathrm{~g}$ was centrally located upon the base plate, along with a 100 g weight. After a total of 10 minutes, the weight was removed. The minimum and maximum diameter of the material were assessed using a digital calliper with a $0.01 \mathrm{~mm}$ precision (Duratool, CPC Farnell, Preston, UK). If the variation was within $1 \mathrm{~mm}$, then the mean diameter was calculated; otherwise the experiment was repeated. The TCS mixed at a water: powder ratio of 0.35 served as a control.

\subsection{Phase analyses}

For phase determination, X-ray diffraction analysis was performed. Materials mixed either with the 0.35 water: powder ratio or the adjusted ratio as determined by the rheometer were immersed in Hank's balanced salt solution (HBSS; Sigma Aldrich, Gillingham, UK) and incubated for 7 days at $37^{\circ} \mathrm{C}$ (Hybaid, Thermo Scientific, Thermo Scientific, Waltham, MA, USA). Subsequently, specimens were vacuum desiccated and crushed using an agate mortar and pestle. The diffractometer (Bruker D8 Advance, Bruker Corp., Billerica, MA, USA) with a CuKa radiation at $40 \mathrm{~mA}$ and $45 \mathrm{kV}$ was set to rotate between $15-50^{\circ}$ with a $0.02^{\circ} 2 \theta$ step and a step time of $0.6 \mathrm{~s}$. Phase identification was undertaken using a search-match software using the ICDD database (International Centre for Diffraction Data, Newtown Square, PA, USA).

\subsection{Ion release assessment}

Analysis of calcium leaching was performed using inductively coupled plasma-optical emission spectrometry (ICP-OES; Optima 8000 ICP-OES, Perkin Elmer, Waltham, MA, USA). Materials were prepared as described above, weighed ( $0.0001 \mathrm{~g}$ precision) and immersed in 
vials containing $5 \mathrm{~mL} \mathrm{HBSS}$ at $37^{\circ} \mathrm{C}$. After 7 days, pellets were retrieved from the vials and the solutions were analysed for calcium using pure HBSS as the blank sample and 6 calibration solutions. Results were assessed in respect to the weight of the material and the solution volume and expressed in $\mu \mathrm{g} / \mathrm{g}$ using the following equation:

Amount of calcium released per weight of pellet $(\mu \mathrm{g} / \mathrm{g})=$ amount of leachate per litre $\left(\frac{\mathrm{mg}}{\mathrm{L}}\right) \times$ volume of solution $(\mathrm{L}) \times 1,000$

weight of cement pellet (g)

\subsection{Scanning electron microscopy (SEM) and X-ray energy dispersive analysis (EDX)}

Materials mixed with the adjusted water: powder ratios were prepared in 15-mm rubber rings (3-mm thick) and allowed to set for $24 \mathrm{~h}$ at $37^{\circ} \mathrm{C}$ in relative humidity. Following vacuum desiccation for 24 hours, specimens were embedded in resin (EpoFix, Struers Ltd, Catcliffe Rotherham, UK) in cylindrical plastic moulds. A grinder/polisher (Phoenix Beta, Buehler, Coventry, UK) was used to grind the materials with progressively finer discs under water or appropriate lubricant irrigation (Diapro and OP-S, Struers Ltd, Catcliffe Rotherham, UK). Consequently, moulds were mounted on aluminium stubs, gold sputter coated (K550X Sputter Coater, Quorum Technologies Ltd, Kent, UK) and viewed under the SEM (EVO MA10, Carl Zeiss Ltd, Cambridge, UK) in backscatter mode at a range of magnifications. EDX analysis was performed at determined points (particles) or bigger fields.

\subsection{Radio-opacity evaluation}

Materials mixed with the adjusted water: powder ratio were evaluated for radioopacity values according to ISO 6876:2012 instructions [31]. Specimens were prepared in rubber discs measuring $10 \pm 1 \mathrm{~mm}$ in diameter and $1 \pm 0.1 \mathrm{~mm}$ thick and allowed to set for 24 hours at $37^{\circ} \mathrm{C}$ in humidity. Specimens were arranged on a photo-stimulable phosphor plate (DenOptix, Gendex Dental Systems, Hatfield, PA, USA) adjacent to a calibrated 
aluminium step wedge (Everything X-ray, High Wycombe, UK) with $3 \mathrm{~mm}$ increments. A standard X-ray machine (Progeny Dental, Midmark Corp, Kettering, OH, USA) was used to irradiate the specimens using an exposure time of $0.80 \mathrm{~s}$ at $10 \mathrm{~mA}$, tube voltage at $65 \pm 5 \mathrm{kV}$ and a cathode-target film distance of $300 \pm 10 \mathrm{~mm}$. The radiographs were then processed (Clarimat 300, Gendex Dental Systems, Hatfield, PA, USA) and a digital image of the radiographs was obtained. For interpretation of results, a method previously described by Formosa et al. was used [32]. Briefly, an imaging programme, ImageJ (Rasband WS, ImageJ; US National Institute of Health, Bethesda, MD, USA) was utilised to calculate the gray pixel value on the radiograph of each step in the step-wedge. Consequently, data for the thickness of the aluminium against the gray pixel value on the radiograph was plotted; the best-fit logarithmic trend line was then identified.

\subsection{Statistical analyses}

Data were evaluated using the Statistical Package for the Social Sciences software version 24 (IBM SPSS Statistics, IBM Corp, Armonk, NY, USA). For the rheological assessment, Dunnett's test using the PC_0.35 as a control was conducted for viscosity values with similar shear rate applied ( 1 decimal precision) between 1 to $20 \mathrm{~s}^{-1}$. The mean values of viscosity of each material were also compared. Independent sample t-tests between materials of the same components and with different ratios were conducted; for the flowability tests and calcium leaching, Dunnett's test using the TCS_0.35 as a control were conducted additionally. For the radio-opacity assessment, one-way analysis of variance and Tukey's multiple comparison tests were performed. The significance level of $p$ $=0.05$ was used for analyses.

\section{Results}




\subsection{Rheological adjustment}

The water: powder ratios of the different formulations in comparison to the one established for Portland cement mixed at a water: powder ratio of 0.35 are shown in Table 1. Both calcium phosphate and the micro-silica increased the water demand of the mixture. The replacement of the different radiopacifiers did not have the same effect. The zirconium oxide did not affect the water: powder ratio required while the tantalum oxide and calcium tungstate increased and decreased the water: powder ratios, respectively. It was also shown that replacement of each cement by inclusion of $30 \%$ radiopacifier resulted in a standard change in the water amount needed to hydrate the material, regardless of the composition of the cement, except for the zirconium oxide which had no effect in the water: powder ratio. These data are shown in Table 2 where the percentage increase or decrease in the water demand is provided.

\subsection{Flowability tests}

Table 3 shows results and comparisons between flowability of materials with the same components but different water: powder ratios, as well as comparisons with the TCS. The inclusion of calcium phosphate increased significantly the flowability of the materials in all the material combinations tested following the water: powder ratio adjustment, regardless of the radiopacifier used $(\mathrm{p}<0.05)$. Calcium phosphate incorporation increased the water demand substantially as indicated in the rheology testing. Consequently, flowability was reduced for CaP- containing materials mixed with the 0.35 water: powder ratio in comparison with the pure TCS $(p<0.05)$, except for the TCS-CaP/CaWO. The incorporation of calcium tungstate resulted in a relatively low water demand as indicated in the rheology testing (Table 2). Thus it compensated for the high water demand of the calcium phosphate in the mixture. 
The micro-silica altered the flowability characteristics of the materials when the adjusted water: powder ratio was applied $(p<0.05)$, except for the TCS-mS10/ZrO-TaO and TCS-mS20/CaWO, where no significant change was reported ( $p>0.05)$.

The different radiopacifiers incorporated in the pure tricalcium silicate did not affect the flowability values of cements in comparison with the non-radiopacified control (TCS_0.35) at both water: powder ratios tested $(p>0.05)$. The incorporation of zirconium oxide and calcium tungstate did not change the flowability characteristics following the adjustment of the water amount used to mix them, however tantalum oxide incorporation increased the material flowability at the adjusted water: powder ratio indicated comparing with the standard 0.35 ratio. The tantalum oxide replacement increased the water demand substantially as shown in Table 2 for the rheology study.

\subsection{X-Ray diffraction analysis}

Results from the XRD analysis are presented in Figure 1a-d. All materials exhibited peaks for calcium hydroxide (ICDD: 01-078-0315). The tricalcium silicate had completely reacted after 7 days and the only peaks visible in the trace were those of the calcium hydroxide at 18 and $34^{\circ} 2 \theta$. The micro-silica was amorphous and no peaks were detected in the respective materials (Figure $1 \mathrm{c}$ and $1 \mathrm{~d}$ ). The radiopacified materials exhibited peaks for zirconium oxide (ICDD: 00-037-1484), tantalum oxide (ICDD: 01-081-8067) and calcium tungstate (ICDD: 04-007-2671) depending on the respective radiopacifiers used. The use of tantalum oxide as radiopacifier enhanced the formation of crystalline calcium hydroxide in the materials with adjusted water: powder ratios (Figure 1a). This increase was also evident when calcium phosphate (Figure 1b) was included but not in the $10 \%$ micro-silica addition. When $20 \%$ micro-silica was added the formation of crystalline calcium hydroxide was 
enhanced with the mixture of zirconium oxide with or without tantalum oxide and the calcium tungstate radiopacifiers (Figure 1d).

\subsection{Ion release assessment}

The calcium ion leaching data is presented in Figure 2. All the materials tested leached relatively high levels of calcium ions in solution after 7 days of immersion in HBSS. The reduction in the water added to the calcium tungstate radiopacified material resulted in a reduction in calcium ion leaching. Increased calcium ion leaching was shown for the additions of calcium phosphate with both zirconium oxide and the combination of zirconium oxide and tantalum oxide. The micro-silica addition did not affect the calcium ion release significantly except for the $20 \%$ addition with the tantalum oxide radiopacifier where a relatively higher calcium ion leaching pattern was observed in the adjusted water: powder ratio. Compared with the cement containing no radiopacifier, the zirconium oxide radiopacified TCS and the materials with additions of micro-silica and tantalum oxide mixed with the 0.35 ratio, released less calcium $(p<0.05)$. Only the material with $20 \%$ microsilicaaddition and tantalum oxide presented significantly higher calcium release, following the adjustment of the water: powder proportion.

\subsection{SEM-EDX characterization}

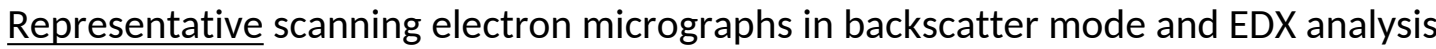
of specific microstructures are presented in Figure 3. The prototype cements were all based on tricalcium silicate with different radiopacifiers replacing them by $30 \%$. Calcium phosphate monobasic and micro-silica were also used as replacement materials. The cement particles in the pure TCS prototype cement exhibited a halo of hydration product surrounding unhydrated cement particles of a similar size in a dense matrix (Figure 3a). Some of the cement particles appeared fully hydrated and the purity of tricalcium silicate 
was verified by the EDX analysis. The addition of the calcium phosphate monobasic changed the hydration kinetics of the tricalcium silicate cement. The calcium phosphate cement particles of the TCS-CaP based materials consisting of calcium, phosphorus and silicon was evident in the micrographs by large halo rims surrounding the unhydrated particles (Figure 3b). The addition of micro-silica also modified the hydration kinetics. In the $10 \%$ replacement (Figure 3c), a dense matrix of hydrated cement particles was evident. In the $20 \%$ replacement specimen (Figure 3d), fewer unhydrated cement particles were apparent in the micrograph. The matrix was densely filled with micro-silica particles.

Different radiopacifier particles and a combination of them ( $\mathrm{ZrO}$ and $\mathrm{TaO}$ ) were included in each cement. All of them appeared well distributed throughout the bulk of each material. Zirconium particles appeared round in morphology and exhibited a range of sizes (Figure 3c). The radiopacifier particles composed of tantalum oxide presented as a relative smaller size while they were occasionally organized in high proximity to each other (Figure 3a). In the materials consisting both of both zirconium and tantalum oxide, similar findings were evident (Figure 3b). Finally, the radiopacifier particles in the calcium tungstate containing prototypes, were spherical in morphology and relatively large in size (Figure $3 \mathrm{~d}$ ). The elemental analysis undertaken on each radiopacifier particle, verified its constituents.

\subsection{Evaluation of radio-opacity}

All materials exhibited acceptable radio-opacity ( $\geq 3 \mathrm{~mm}$ aluminium) after the adjustment of the water: powder ratio as specified by ISO 6876:2012 [31] (Table 4). The micro-silica addition with calcium tungstate and the $10 \%$ micro-silica with zirconium oxide, generated higher values of radio-opacity, with significant differences compared with the majority of the other materials, especially those containing tantalum oxide $(p<0.05)$. No statistically significant differences were identified between the other materials tested $(p>$ 
0.05).

\section{Discussion}

The use of a rheometer has been proposed by several previous studies for the assessment of rheological properties of endodontic sealers [33, 34], impression materials [35], composites [36-39], as well as for determining the material setting time [40]. For endodontic sealers [41, 42], the materials are loaded between two parallel plates and different shear strain tests can be applied. The change in viscosity values as a result of the deformation of the material can thus be monitored. In the present study, a rheometer was used to determine viscosity values for increasing values of shear rate for each prototype material with different additives tested. Portland cement mixed with a 0.35 water: powder ratio was used as a control, since this mixture has the most acceptable handling properties for clinical use and the process of its hydration reaction has been extensively assessed [11]. A standard amount of water was added to each test material to render them adequately flowable and ensure reproducibility of the experiments (Table 2). This was considered important since the maximum loading force applied by the upper plate was not adequate enough to achieve an even contact surface throughout the mixture due to the cement's stiffness.

Although a 2-minute flow-sweep test was initially designed, it was noted that viscosity values for a shear rate above $20 \mathrm{~s}^{-1}$ could result in separation of the material with the upper plate and they were thus not significant for evaluation. Therefore, results were assessed for the first 23 seconds of the experiment. Data obtained from the rheological assessments showed an increase in the water demand when calcium phosphate, micro-silica or tantalum oxide was incorporated into the material. Replacement with zirconium oxide did not affect the water amount and resulted in similar viscosity values compared with the 
unmodified cement, while calcium tungstate decreased the water demand. Apart from zirconium oxide, a standard percentage change of the water: powder ratio was determined when a $30 \%$ replacement of the radiopacifiers was incorporated compared with the unradiopacified cement. Thus, the hypothesis that different compounds will not alter the water demand of the cement was rejected.

ISO 6876:2012 flowability tests were also performed [31]. Results from the compression glass plate analyses demonstrated a significant change in flowability values of most prototype materials after the adjustment of the water: powder ratio, especially for the calcium phosphate and micro-silica containing cements $(p<0.05)$. However, comparisons with the unradiopacified cement were only in partial agreement with results obtained from the rheometer. Therefore, the pattern in the adjustment of the water: powder ratio could not be supported in all cases. This can partially be attributed to the lower sensitivity of the compression glass plate test [33]. ISO flowability tests provide information on the compressed diameter of the material after a specific amount of load is applied. In contrast, the protocol design of the rheological adjustment is dynamic; apparent viscosity values are calculated as progressively higher shear strain is applied to the material. Moreover, the ISO flowability tests are designed for endodontic sealers; such materials are expected to present relatively higher flowability compared with hydraulic cements [31]. To overcome these limitations, a new method using micro-computed tomography has also been proposed recently for reproducible flowability assays of root-repair materials [25]. This model is applied in 3-dimensions and thus potentially more relevant to the clinical scenario.

X-ray diffraction was used for crystalline phase analysis. All materials formed calcium hydroxide while the peaks for tricalcium silicate were at reduced intensity indicated that a significant part of the hydration reaction had occurred by 7 days. The adjustment of the 
water: powder ratio resulted in differences in the calcium hydroxide peaks mainly for the tantalum containing materials, where increase of the water amount included was correlated with higher intensity peaks. No differences in the diffraction patterns where detected in the $10 \%$ micro-silica replacement. Micro-silica affects material hydration and calcium ion release in the long term by formation of calcium silicate hydrate from the reaction of the calcium hydroxide and the silicon oxide [43]. This is very well documented in the construction industry and even exploited to enhance the strength of concrete mixtures in the long term [44]. The current testing was performed after 7 days so the effect of the micro-silica on the calcium ion release may have been masked. Calcium leaching in solution is considered an important parameter for endodontic materials as it has been correlated with the beneficial biological properties of the hydraulic cements, such as positively promoting pulp tissue responses and dentin bridge formation [45-47]. The increase of the water: powder ratio resulted in higher calcium release and this is in accordance with a previous study [24]. The material characterization was performed by SEM and EDX using the back-scatter detector after the adjustment of the water: powder proportion in order to monitor the hydration reaction. The hydration mechanism was altered after the addition of calcium phosphate and micro-silica as is evidenced by the micrographs. The different radiopacifiers were organized throughout the bulk of the material or spread as independent particles, especially following calcium tungstate inclusion.

Finally, we evaluated the radio-opacity of the materials after modifying the water amount. All the materials were adequately radiopaque after modification of the water amount according to ISO 6876:2012 specifications [31]. Notably it has been previously shown that an increase in the water: powder ratio is negatively correlated with the radioopacity of hydraulic cements [24]. 
Results from the physicochemical analyses indicated that the null hypothesis regarding the modification of material's properties following the change in the hydraulic cement's water demand can be partially rejected. The effect of different amounts of water on the physical properties of Portland cement has been described previously [30]. Therefore, it should always be taken into consideration when evaluation of physicochemical properties of TCS-based cements is carried out after addition of different compounds and radiopacifiers. As a consequence, a potential alteration in characteristics could be incorrectly attributed to the additives. It is also evident that the suggested powder to liquid proportions suggested by the manufacturer, should be strictly followed.

\section{Conclusions}

The present study provides a reproducible method for the calculation of the water amount in hydraulic cements by establishing similar rheological properties using a reference material. To the best of our knowledge, no other protocol for this adjustment exists. The water demand for materials which include radiopacifiers and additives varies depending on the type of radiopacifier and additive. It is important to adjust the water: powder ratio based on the radiopacifier and additive type as this will affect the material properties.

\section{Acknowledgments}

Gabor Dravavolgyi and Jianguo Liu for their technical assistance in rheology and scanning electron microscopy respectively.

\section{References}

[1] Torabinejad M, White DJ. Tooth filling material and method of use. United States patent US 5415547; May 16, 1995. 
[2] Chong BS, Pitt Ford TR, Hudson MB. A prospective clinical study of mineral trioxide aggregate and IRM when used as root-end filling materials in endodontic surgery. Int Endod J 2003;36:520-6.

[3] Torabinejad M, Parirokh M, Dummer PMH. Mineral trioxide aggregate and other bioactive endodontic cements: an updated overview - part II: other clinical applications and complications. Int Endod J 2018;51:284-317.

[4] Torabinejad M, Chivian N. Clinical applications of mineral trioxide aggregate. J Endod 1999;25:197-205.

[5] Parirokh M, Torabinejad M, Dummer PMH. Mineral trioxide aggregate and other bioactive endodontic cements: an updated overview - part I: vital pulp therapy. Int Endod J 2018;51:177-205.

[6] Schembri M, Peplow G, Camilleri J. Analyses of heavy metals in mineral trioxide aggregate and Portland cement. J Endod 2010;36:1210-15.

[7] Camilleri J, Kralj P, Veber M, Sinagra E. Characterization and analyses of acidextractable and leached trace elements in dental cements. Int Endod J 2012;45:737-43.

[8] Monteiro Bramante C, Demarchi AC, de Moraes IG, Bernadineli N, Garcia RB, Spångberg LS, Duarte MA. Presence of arsenic in different types of MTA and white and gray Portland cement. Oral Surg Oral Med Oral Pathol Oral Radiol Endod. 2008;106:909-13.

[9] Camilleri J. Characterization of hydration products of mineral trioxide aggregate. Int Endod J 2008;41:408-17.

[10] Marciano MA, Costa RM, Camilleri J, Mondelli RF, Guimaraes BM, Duarte MA. Assessment of color stability of white mineral trioxide aggregate angelus and bismuth oxide in contact with tooth structure. J Endod 2014;40:1235-40.

[11] Camilleri J. Characterization and hydration kinetics of tricalcium silicate cement for use as a dental biomaterial. Dent Mater 2011;27:836-44.[12] Camilleri J, Sorrentino F, Damidot D. Investigation of the hydration and bioactivity of radiopacified tricalcium silicate cement, Biodentine and MTA Angelus. Dent Mater 2013;29:580-93.

[13] Camilleri J. Characterization and chemical activity of Portland cement and two experimental cements with potential for use in dentistry. Int Endod J 2008;41:791-9. [14] Grech L, Mallia B, Camilleri J. Characterization of set Intermediate Restorative Material, Biodentine, Bioaggregate and a prototype calcium silicate cement for use as rootend filling materials. Int Endod J 2013;46:632-41.

[15] Gandolfi MG, Spagnuolo G, Siboni F, Procino A, Rivieccio V, Pelliccioni GA, et al. Calcium silicate/calcium phosphate biphasic cements for vital pulp therapy:

chemicalphysical properties and human pulp cells response. Clin Oral Invest 2015;19:207589.

[16] Cutajar A, Mallia B, Abela S, Camilleri J. Replacement of radiopacifier in mineral trioxide aggregate; characterization and determination of physical properties. Dent Mater 2011;27:879-91.

[17] Brasseler USA [Internet]. BC Sealer safety data sheet [cited 201827 September]. Available from: http://brasselerusa.com/wp-content/files/SDS0003\%20TotalFill\%20BC\%20Sealer\%20SDS\%20\%20REV\%20B.pdf.

[18] Khalil I, Naaman A, Camilleri J. Properties of tricalcium silicate sealers. J Endod 2016;42:1529-35.

[19] Kebudi Benezra M, Schembri Wismayer P, Camilleri J. Interfacial characteristics and 
cytocompatibility of hydraulic sealer cements. J Endod 2018;44:1007-17.

[20] Akbari M, Zebarjad SM, Nategh B, Rouhani A. Effect of nano silica on setting time and physical properties of mineral trioxide aggregate. J Endod 2013;39:1448-51.

[21] Viapiana R, Guerreiro-Tanomaru JM, Hungaro-Duarte MA, Tanomaru-Filho M, Camilleri J. Chemical characterization and bioactivity of epoxy resin and Portland cementbased sealers with niobium and zirconium oxide radiopacifiers. Dent Mater 2014;30:1005-20.

[22] Schembri-Wismayer P, Camilleri J. Why Biphasic? Assessment of the effect on cell proliferation and expression. J Endod 2017;43:751-9.

[23] Fridland M, Rosado R. Mineral trioxide aggregate (MTA) solubility and porosity with different water-to-powder ratios. J End 2003;29:814-7.

[24] Cavenago BC, Pereira TC, Duarte MAH, Ordinola-Zapata R, Marciano MA, Bramante $\mathrm{CM}$, et al. Influence of powder-to-water ratio on radiopacity, setting time, $\mathrm{pH}$, calcium ion release and a micro-CT volumetric solubility of white mineral trioxide aggregate. Int Endod J 2014;47:120-6.

[25] Tanomaru-Filho M, Torres FFE, Bosso-Martelo R, Chavez-Andrade GM, Bonetti-Filho I, Guerreiro-Tanomaru JM. A Novel model for evaluating the flow of endodontic materials using micro-computed tomography. J Endod 2017;43:796-800.

[26] Camilleri J. Evaluation of selected properties of mineral trioxide aggregate sealer cement. J Endod 2009;35:1412-7.

[27] Tanomaru-Filho M, Garcia AC, Bosso-Martelo R, Berbert FL, Nunes Reis JM, Guerreiro-Tanomaru JM. Influence of addition of calcium oxide on physicochemical properties of Portland cement with zirconium or niobium oxide. J Conserv Dent 2015;18:105-8.

[28] Zhou HM, Shen Y, Zheng W, Li L, Zheng YF, Haapasalo M. Physical properties of 5 root canal sealers. J Endod 2013;39:1281-6.[29] Silva Almeida LH, Moraes RR, Morgental RD, Pappen FG. Are premixed calcium silicate-based endodontic sealers comparable to conventional materials? A systematic review of in vitro studies. J Endod 2017;43:527-35. [30] Neville AM. Properties of concrete. 5th ed. Essex: Pearson; 2002.

[31] International Standards Organization. Dentistry- root canal sealing materials. ISO 6876;2012.

[32] Formosa LM, Mallia B, Camilleri J. The effect of curing conditions on the physical properties of tricalcium silicate cement for use as a dental biomaterial. Int Endod J 2012;45:326-36.

[33] Chang SW, Lee YK, Zhu Q, Shon WJ, Lee WC, Kum KY, et al. Comparison of the rheological properties of four root canal sealers. Int J Oral Sc 2015;7:56-61.

[34] Lacey S, Pitt Ford TR, Watson TF, Sherriff M. A study of the rheological properties of endodontic sealers. Int Endod J 2005;38:499-504.

[35] German MJ, Carrick TE, McCabe JF. Surface detail reproduction of elastomeric impression materials related to rheological properties. Dent Mater 2008;24:951-6.

[36] Ellakwa A, Cho N, Lee IB. The effect of resin matrix composition on the polymerization shrinkage and rheological properties of experimental dental composites. Dent Mater 2007;23:1229-35.

[37] Beun S, Bailly C, Dabin A, Vreven J, Devaux J, Leloup G. Rheological properties of experimental Bis-GMA/TEGDMA flowable resin composites with various macrofiller/microfiller ratio. Dent Mater 2009;25:198-205. 
[38] Lee I-B, Min S-H, Kim S-Y, Ferracane J. Slumping tendency and rheological properties of flowable composites. Dent Mater 2010;26:443-8.

[39] Kim M-H, Min S-H, Ferracane J, Lee I-b. Initial dynamic viscoelasticity change of composites during light curing. Dent Mater 2010;26:463-70.

[40] Ha WN, Nicholson TM, Kahler B, Walsh LJ. Rheological characterization as an alternative method to indentation for determining the setting time of restorative and endodontic cements. Mater 2017;10(12)1451. https://doi.org/10.3390/ma10121451) [41] Ørstavik D. Physical properties of root canal sealers: measurement of flow, working time, and compressive strength. Int Endod J 1983;16:99-107.

[42] Vermilyea SG, Huget EF, De Simon LB. Extrusion of rheometry of fluid materials. J Dent Res 1979;58:1691-5.

[43] Camilleri J, Montesin FE, Curtis RV, Ford TR. Characterization of Portland cement for use as a dental restorative material. Dent Mater 2006;22:569-75.

[44] Bache HH, Densified cement/ultra-fine particle-based materials. Proceedings of the 2nd International conference on superplasticizers in concrete, 1981 June 10-12; Ottawa, Canada. Aalborg Portland, 1981.

[45] Foreman PC, Barnes IE. Review of calcium hydroxide. Int End J 1990;23:283-97. [46] Tamburic SD, Vuleta GM, Ognjanovic JM. In vitro release of calcium and hydroxyl ions from two types of calcium hydroxide preparation. Int Endod J 1993;26:125-30.

[47] Prati C, Gandolfi MG. Calcium silicate bioactive cements: Biological perspectives and clinical applications. Dental Mater 2015;31:351-70 
Table 1. Adjusted water: powder ratio for Portland cement (PC) with different compounds or radiopacifiers as calculated by the rheological assessment. The numerical scale has been set in two decimal digits.

\begin{tabular}{|c|c|c|c|c|c|}
\hline \multirow[b]{2}{*}{$P C$} & \multirow[b]{2}{*}{$\begin{array}{c}\text { No } \\
\text { radiopacifier }\end{array}$} & \multicolumn{4}{|c|}{$30 \%$ radiopacifier } \\
\hline & & ZrO & $\mathrm{TaO}$ & $\begin{array}{l}\text { ZrO- } \\
\mathrm{TaO}\end{array}$ & CaWO \\
\hline $\begin{array}{c}\text { No } \\
\text { addition }\end{array}$ & $\begin{array}{c}0.35 \\
\text { (control) }\end{array}$ & 0.35 & 0.42 & 0.37 & 0.26 \\
\hline $15 \% \mathrm{CaP}$ & 0.50 & 0.50 & 0.60 & 0.53 & 0.37 \\
\hline $10 \% \mathrm{mS}$ & 0.40 & 0.40 & 0.48 & 0.42 & 0.30 \\
\hline $20 \% \mathrm{mS}$ & 0.50 & 0.50 & 0.60 & 0.53 & 0.37 \\
\hline
\end{tabular}

Table 2. Standard percentage change in the water demand of hydraulic cements after $30 \%$ replacement of radiopacifier.

\begin{tabular}{|c|c|}
\hline $\begin{array}{c}30 \% \\
\text { radiopacifier }\end{array}$ & $\begin{array}{c}\text { Modification in the water: powder } \\
\text { ratio }\end{array}$ \\
\hline ZrO & Same \\
\hline TaO & $20 \% \uparrow$ \\
\hline ZrO-TaO & $5.71 \% \uparrow$ \\
\hline CaWO & $25.71 \% \downarrow$ \\
\hline
\end{tabular}


Table 3. Mean flow values and standard deviation of tested prototype materials mixed with different ratios according to ISO 6876(2012). A = Adjusted; The latin letter ' $a$ ' indicates significant difference in flowability values of materials with the same components after mixing with different water amounts $(p<0.05)$; the asterisk indicates significant difference in flowability values comparing to the TCS_0.35 control $(p<0.05)$.

\begin{tabular}{|c|c|c|c|c|c|}
\hline \multirow[b]{2}{*}{ Material } & \multirow[b]{2}{*}{ Ratio } & \multicolumn{4}{|c|}{$30 \%$ radiopacifier } \\
\hline & & ZrO & $\mathrm{TaO}$ & ZrO-TaO & CaWo \\
\hline \multirow{2}{*}{ TCS } & 0.35 & $9.22 \pm 0.1$ & $8.63 \pm 0.5$ & $9.99 \pm 0.9$ & $10.8 \pm 1.1$ \\
\hline & $A$ & - & $10.91 \pm 0.3^{a}$ & $10.09 \pm 0.6$ & $9.38 \pm 1$ \\
\hline \multirow{2}{*}{ TCS-CaP } & 0.35 & $8.14 \pm 0.04^{*}$ & $6.84 \pm 0.3^{*}$ & $7.06 \pm 0.5^{*}$ & $8.34 \pm 0.4$ \\
\hline & A & $10.45 \pm 0.3^{a}$ & $10.3 \pm 0.5^{a}$ & $10.12 \pm 0.2^{a}$ & $9.17 \pm 0.2^{a}$ \\
\hline \multirow{2}{*}{ TCS-mS10 } & 0.35 & $8.65 \pm 0.3$ & $8.34 \pm 0.5$ & $8.35 \pm 0.4$ & $10.75 \pm 0.2^{c}$ \\
\hline & $A$ & $11.36 \pm 0.3^{* a}$ & $10.72 \pm 1.4^{a}$ & $9.31 \pm 0.5$ & $9.37 \pm 0.6$ \\
\hline \multirow{2}{*}{ TCS-mS20 } & 0.35 & $8.41 \pm 0.2$ & $8.42 \pm 0.3$ & $8.47 \pm 0.4$ & $10.57 \pm 0.3$ \\
\hline & $A$ & $10.57 \pm 0.5^{a}$ & $11.11 \pm 0.4^{*^{a}}$ & $13.97 \pm 0.3^{*^{a}}$ & $10.34 \pm 0.4$ \\
\hline TCS_0. & rol) & \multicolumn{4}{|c|}{$9.65 \pm 0.6$} \\
\hline
\end{tabular}


Table 4. Mean and standard deviation of radio-opacity values of materials ( $\mathrm{mm}$ aluminium) after the adjustment of the water: powder ratio. Latin letters $a, b$ and $c$ indicate statistical significant differences from TCS-mS10/CaWO, TCS-mS10/ZrO and TCS-mS20/CaWO respectively $(p<0.05)$

\begin{tabular}{|c|c|c|c|c|}
\hline \multirow{2}{*}{ TCS } & \multicolumn{4}{|c|}{ 30\% radiopacifier } \\
\cline { 2 - 5 } & ZrO & TaO & ZrO-TaO & CaWO \\
\hline \multirow{2}{*}{ No addition } & $3.6 \pm 0.2$ & $3.1 \pm 0.1^{a b c}$ & $3.5 \pm 0.2^{b}$ & $3.1 \pm 0.1^{a b c}$ \\
\hline $15 \% \mathrm{CaP}$ & $3.1 \pm 0.1^{a b c}$ & $3.1 \pm 0.02^{a b c}$ & $3.3 \pm 0.2^{a b c}$ & $3.5 \pm 0.1^{b}$ \\
\hline $10 \% \mathrm{mS}$ & $4 \pm 0.1$ & $3.3 \pm 0.1^{a b c}$ & $3.6 \pm 0.04$ & $4.2 \pm 0.4$ \\
\hline $\mathbf{2 0} \% \mathrm{mS}$ & $3.1 \pm 0.2^{a b c}$ & $3.2 \pm 0.3^{a b c}$ & $3.3 \pm 0.05^{a b c}$ & $3.9 \pm 0.3$ \\
\hline
\end{tabular}




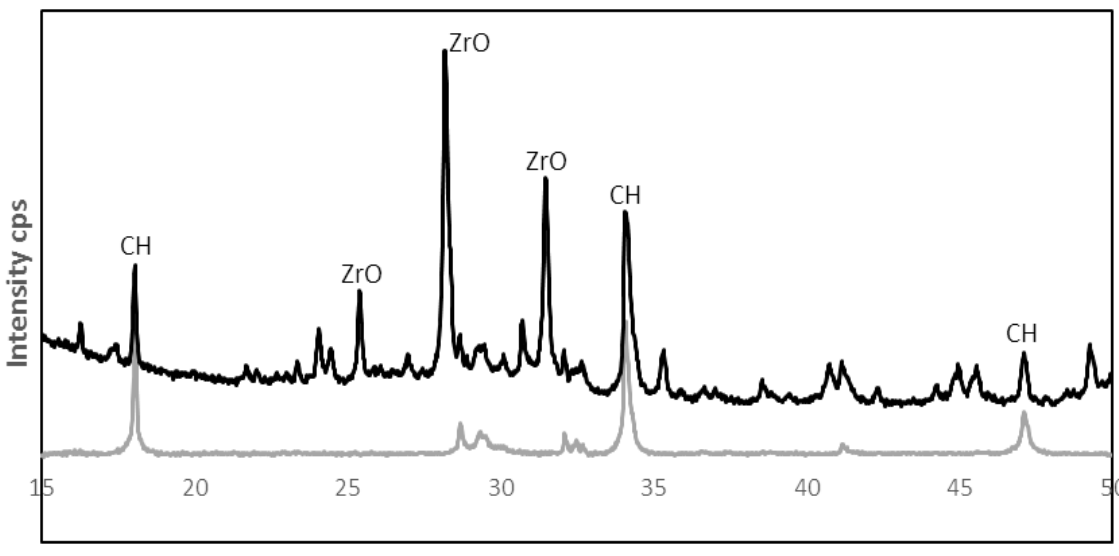

Degrees 2 theta

-TCS_0.35 -TCS/ZrO_0.35

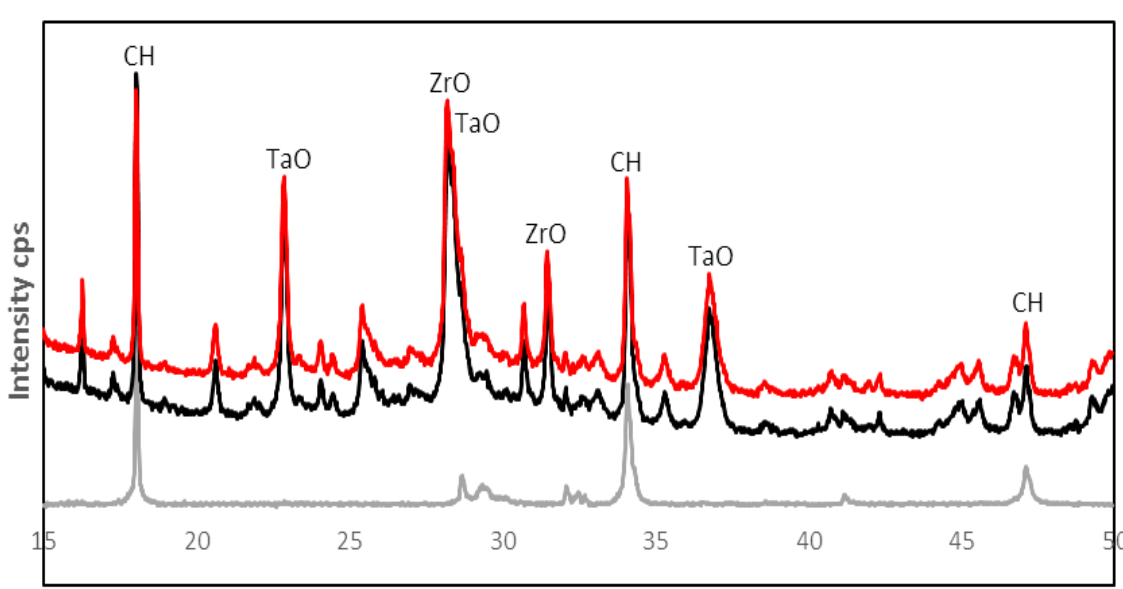

Degrees 2 theta

-TCS_0.35 -TCS/ZrO-TaO_0.35

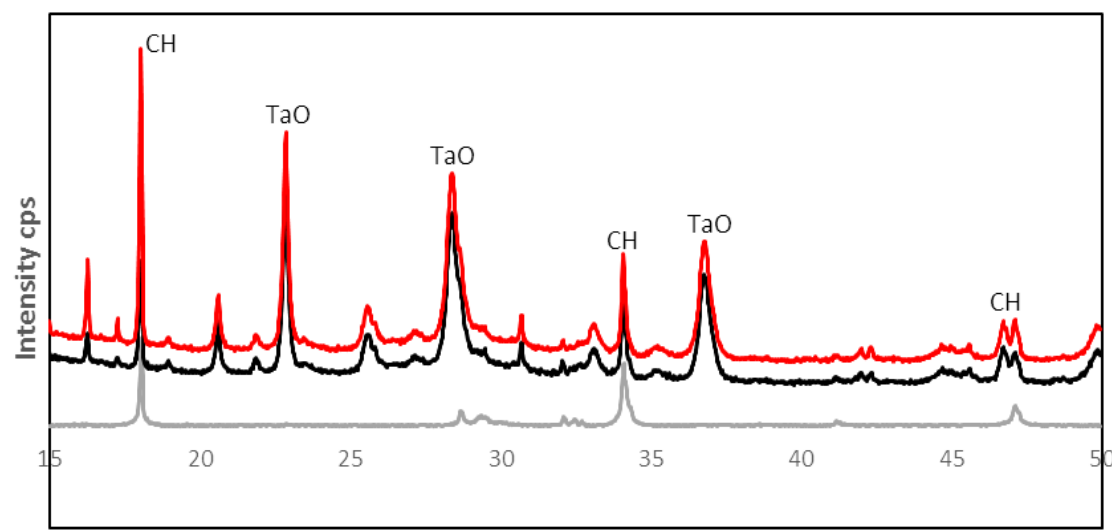

Degrees 2 theta

-TCS_0.35 -TCS/TaO_0.35

- TCS/TaO_A

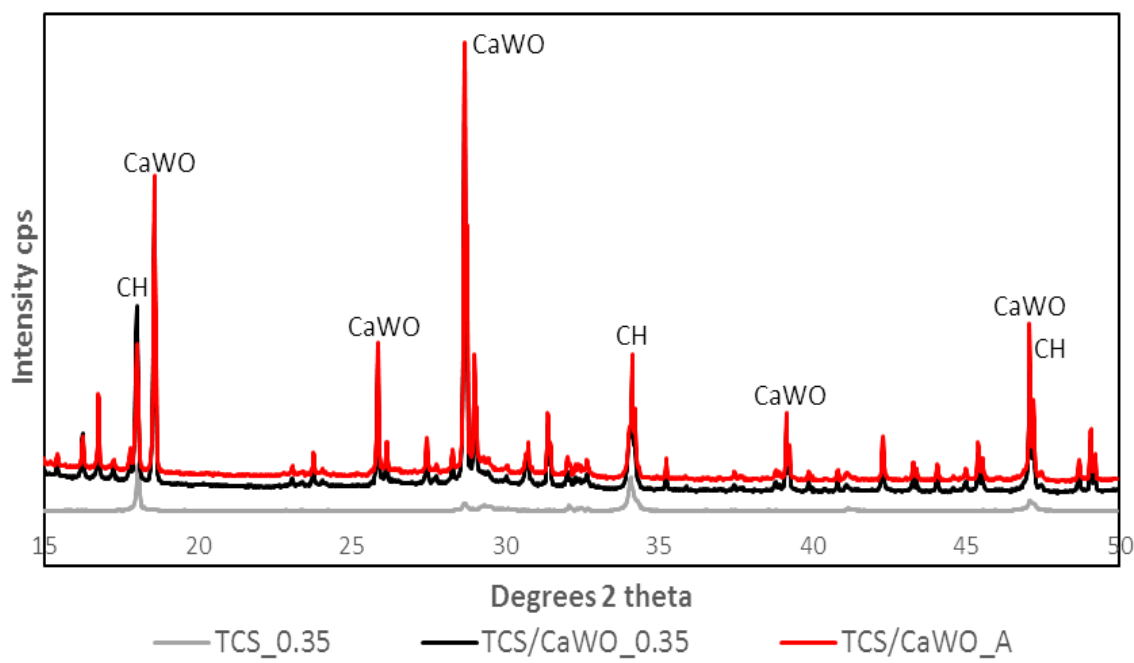

Figure 1a. X-ray diffraction plots of tricalcium silicate cement and test prototype materials replaced with zirconium oxide, tantalum oxide, a mixture of both zirconium oxide and tantalum oxide and calcium tungstate mixed in a 0.35 water: powder ratio or an adjusted ratio after immersion in Hank's balanced salt solution for 1 week. $\mathrm{CH}$ : calcium hydroxide. 


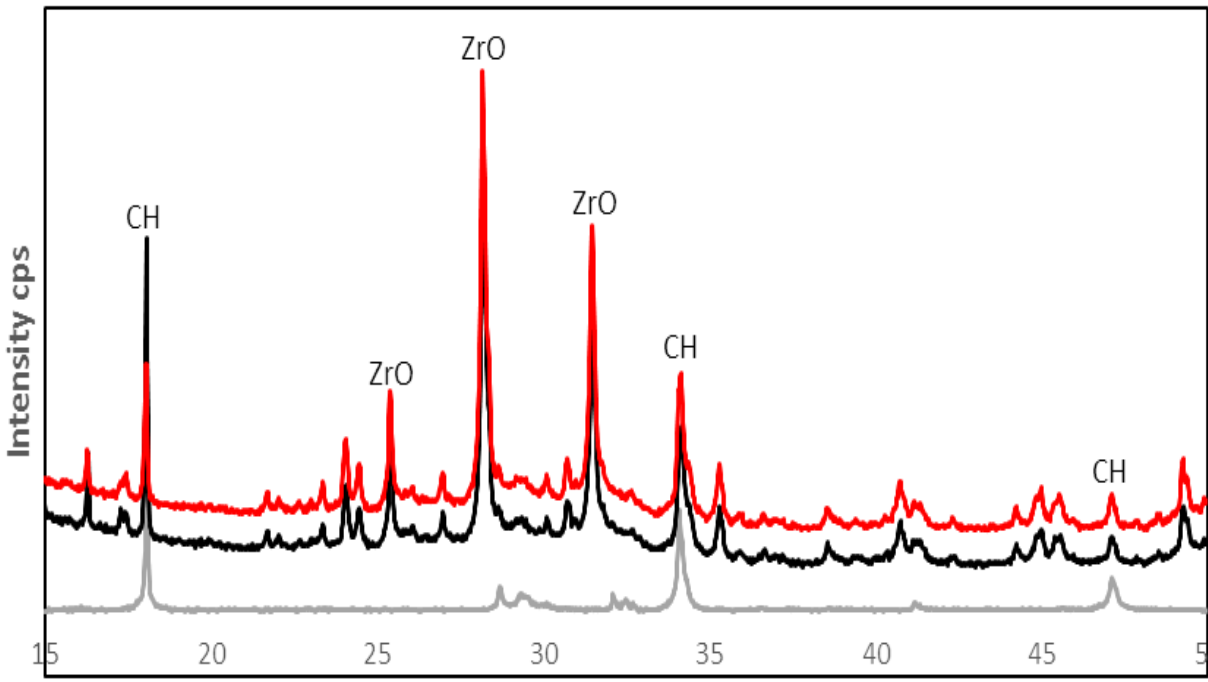

Degrees 2 theta

-TCS_0.35 -TCS-CaP/ZrO_0.35 -TCS-CaP/ZrO_A

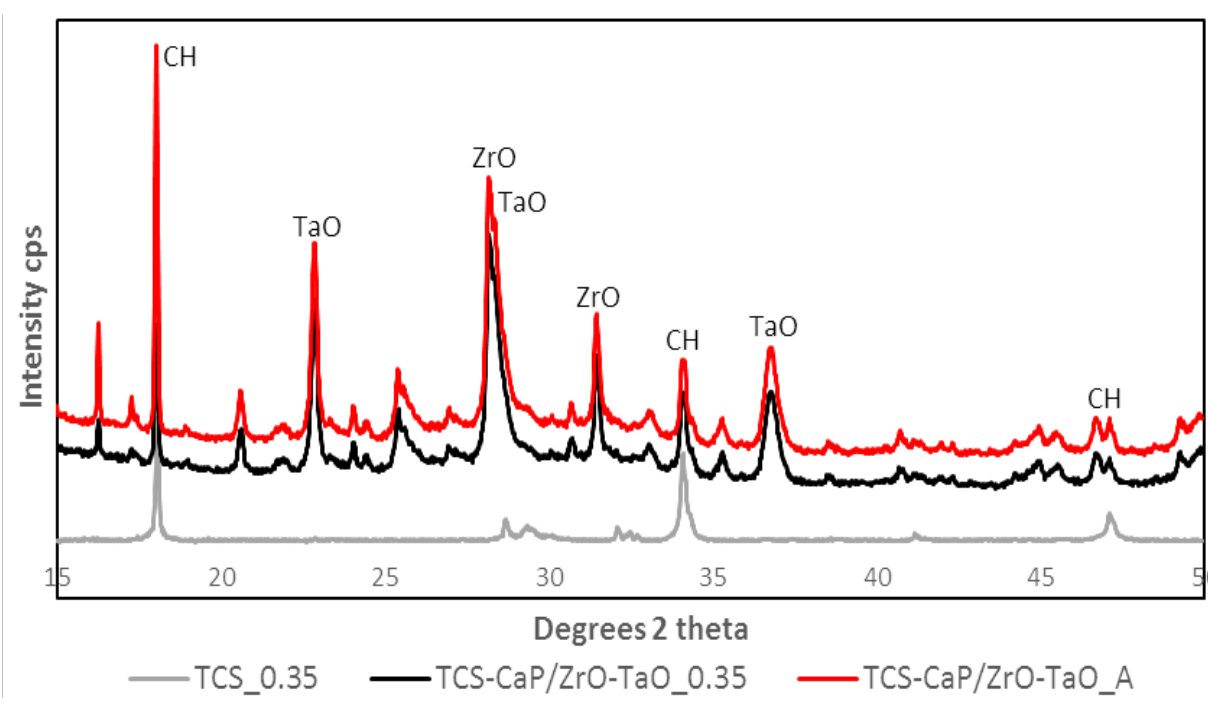

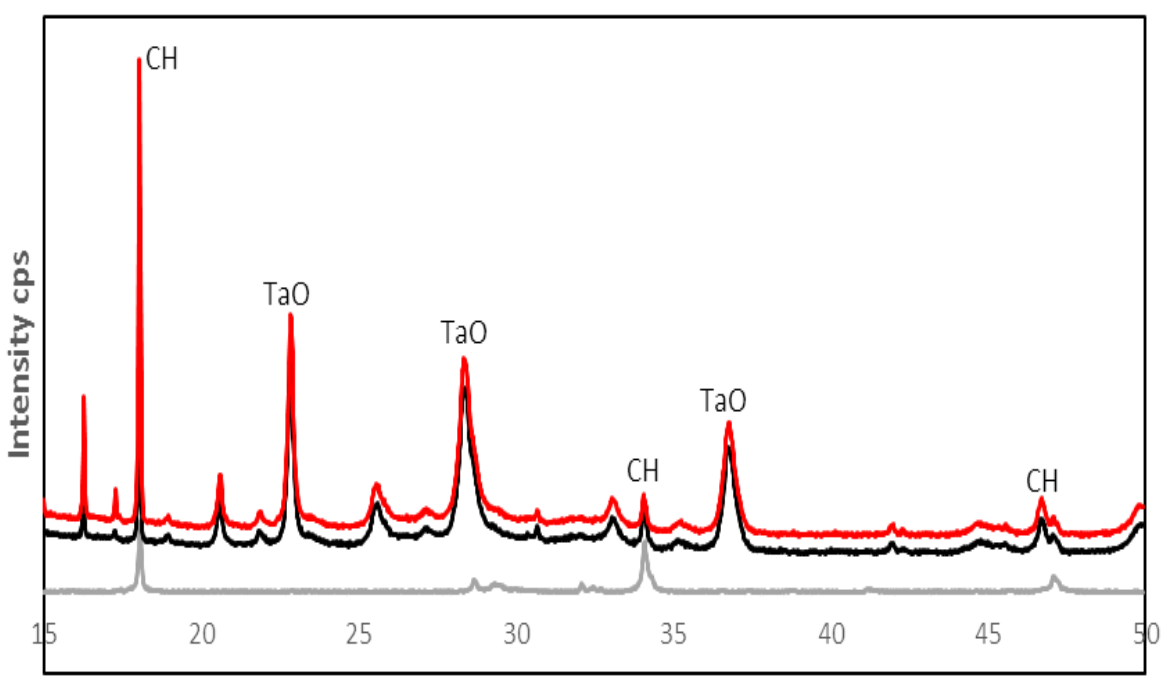

Degrees 2 theta

-TCS_0.35 -TCS-CaP/TaO_0.35 -TCS-CaP/TaO_A

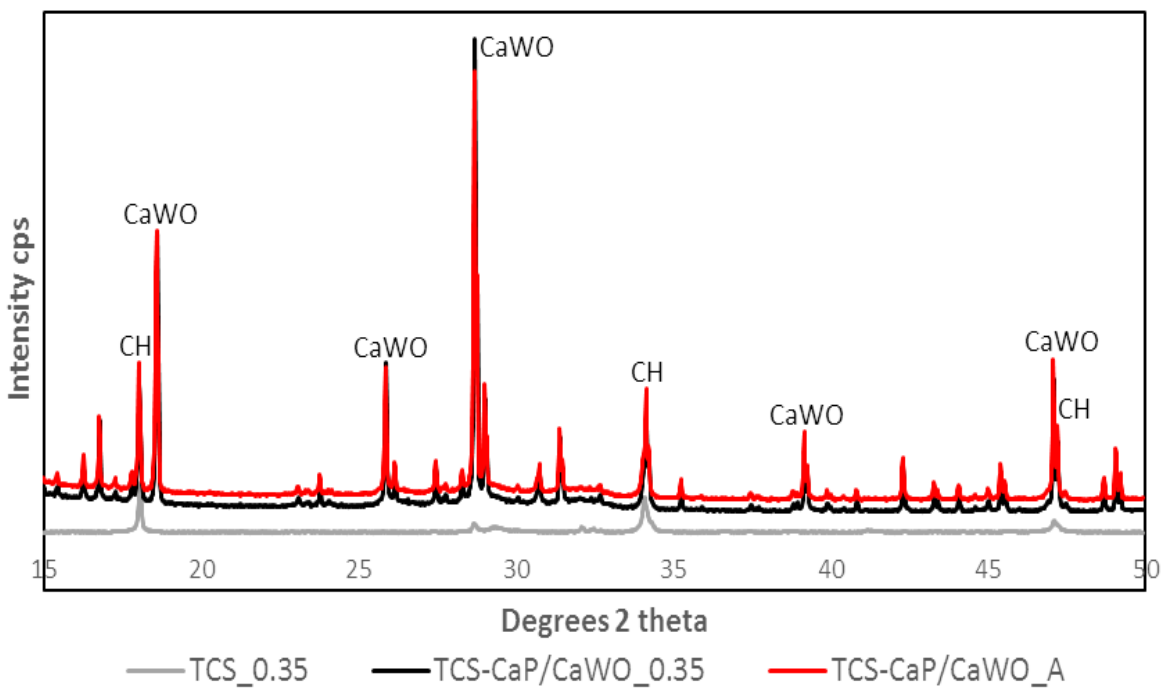

Figure 1 b. X-ray diffraction plots of tricalcium silicate cement and test prototype materials with different radio-opacifiers and incorporating calcium phosphate monobasic mixed in a 0.35 water: powder ratio or an adjusted ratio after immersion in Hank's balanced salt solution for 1 week. $\mathrm{CH}$ : calcium hydroxide. 


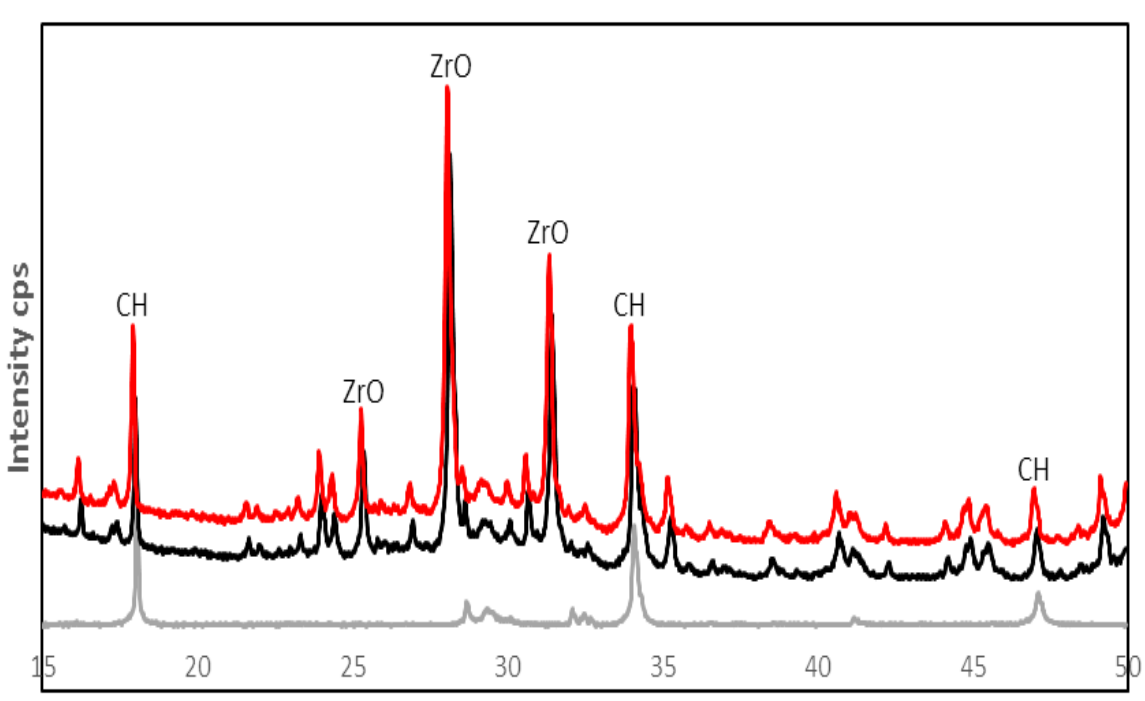

Degrees 2 theta

-TCS_0.35 —TCS-mS10/ZrO_0.35 —TCS-mS10/Zro_A

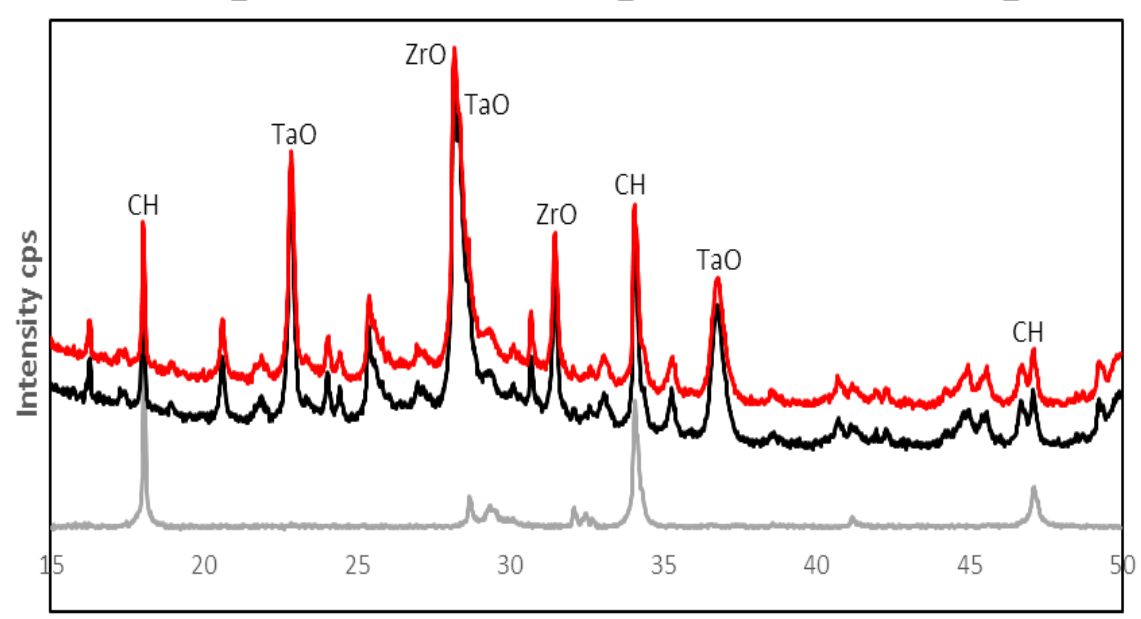

Degrees 2 theta

—TCS_0.35 —TCS-mS10/ZrO-TaO_0.35 —TCS-mS10/ZrO-TaO_A

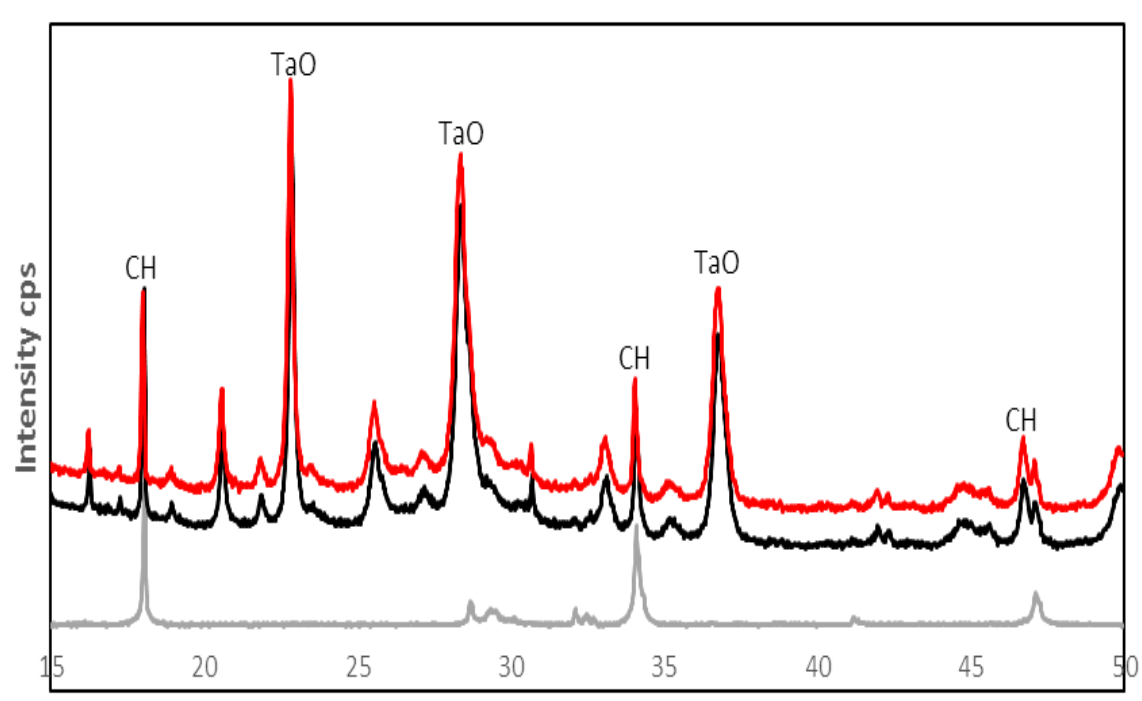

Degrees 2 theta

-TCS_0.35 —TCS-mS10/TaO_0.35 -TCS-mS10/TaO_A

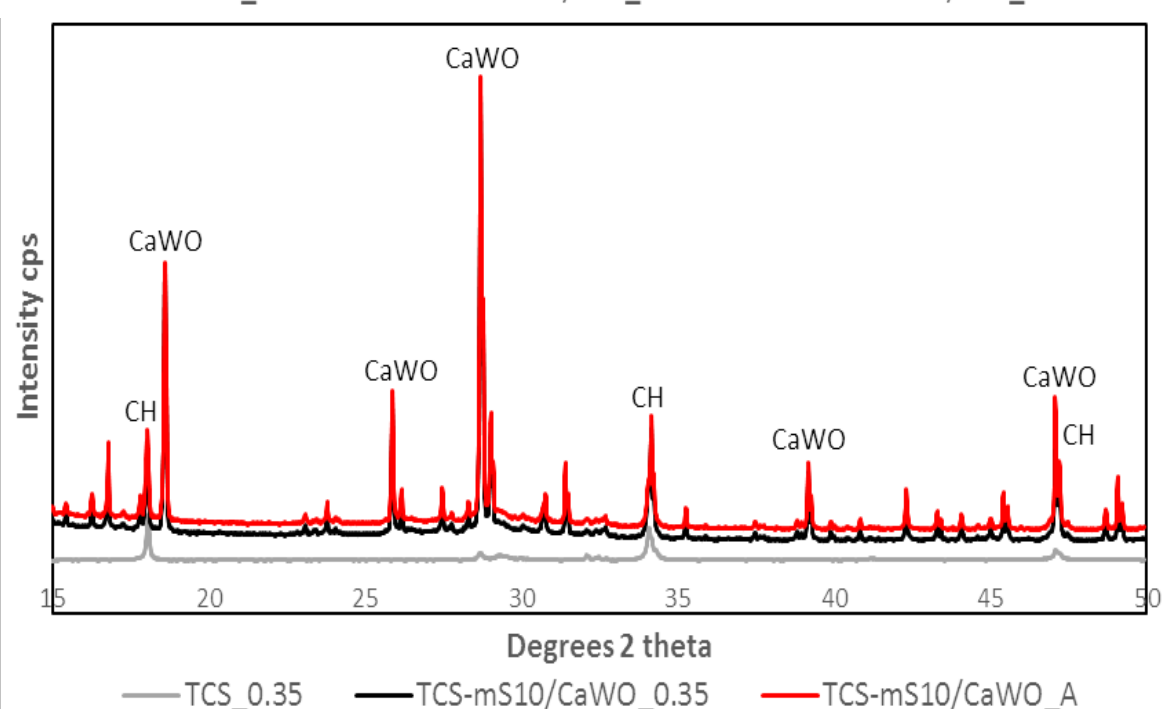

Figure 1c. X-ray diffraction plots of tricalcium silicate cement and test prototype materials with different radio-opacifiers and incorporating $10 \%$ microsilica mixed in a 0.35 water: powder ratio or an adjusted ratio after immersion in Hank's balanced salt solution for 1 week. $\mathrm{CH}$ : calcium hydroxide. 


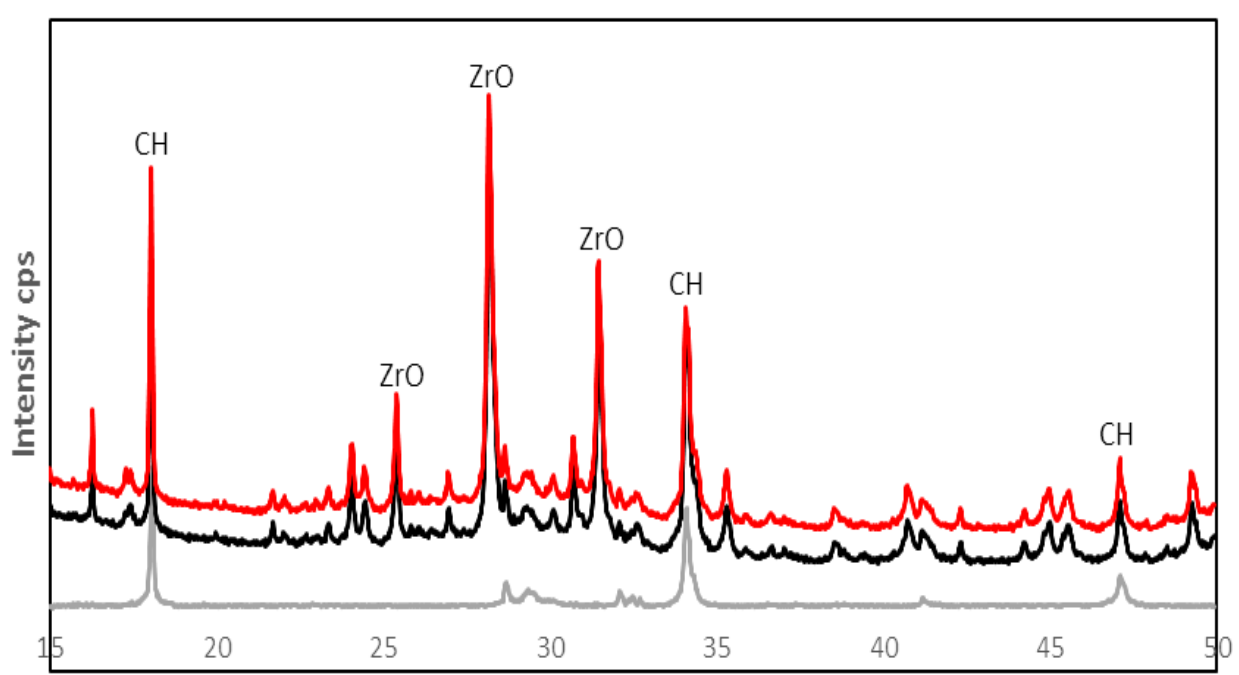

Degrees 2 theta

-TCS_0.35 -TCS-mS20/ZrO_0.35 -TCS-mS20/ZrO_A

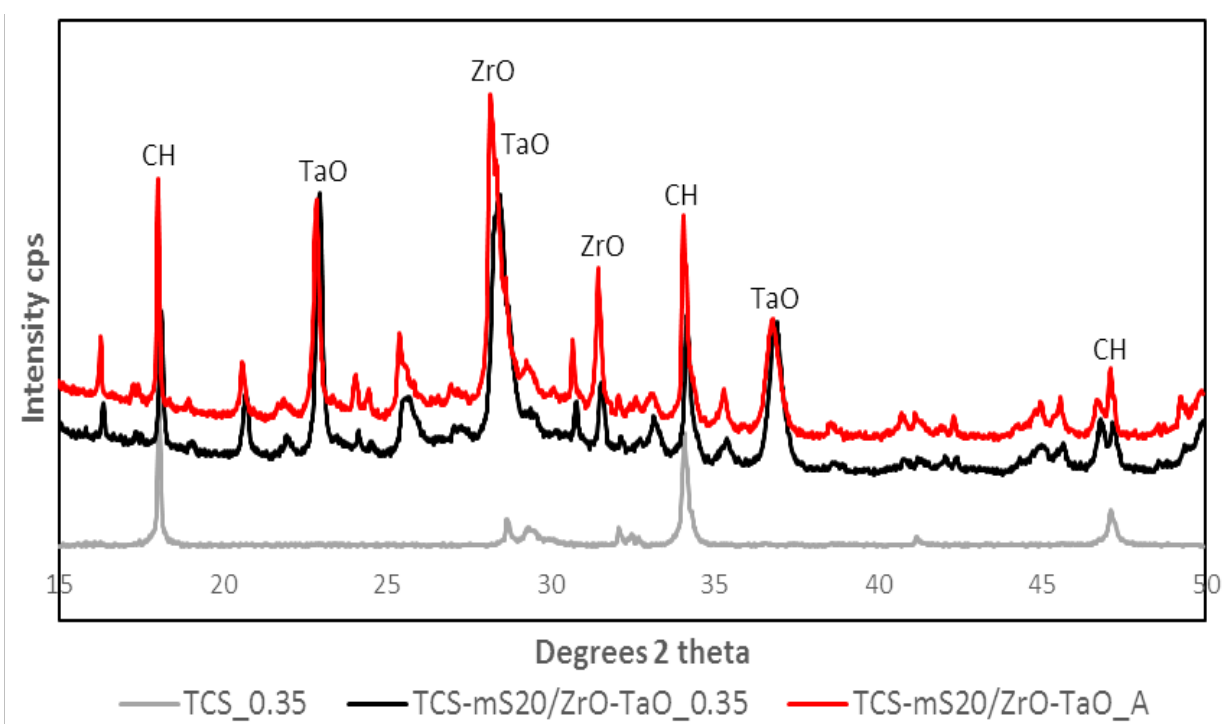

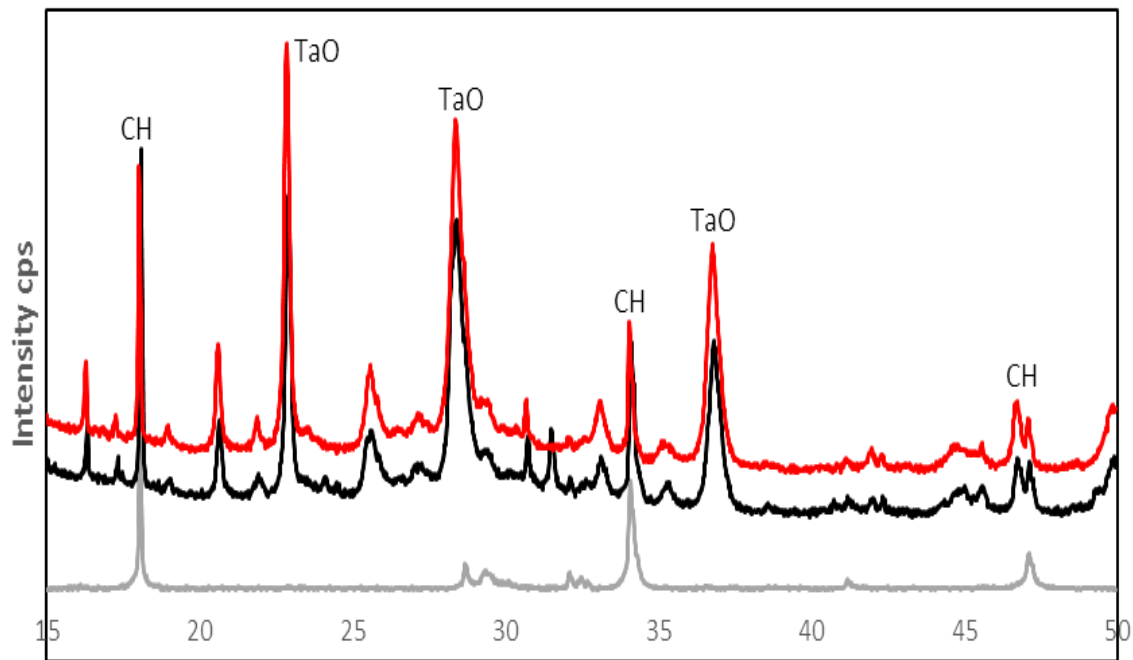

Degrees 2 theta

-TCS_0.35 -TCS-mS20/TaO_0.35 -TCS-mS20/TaO_A

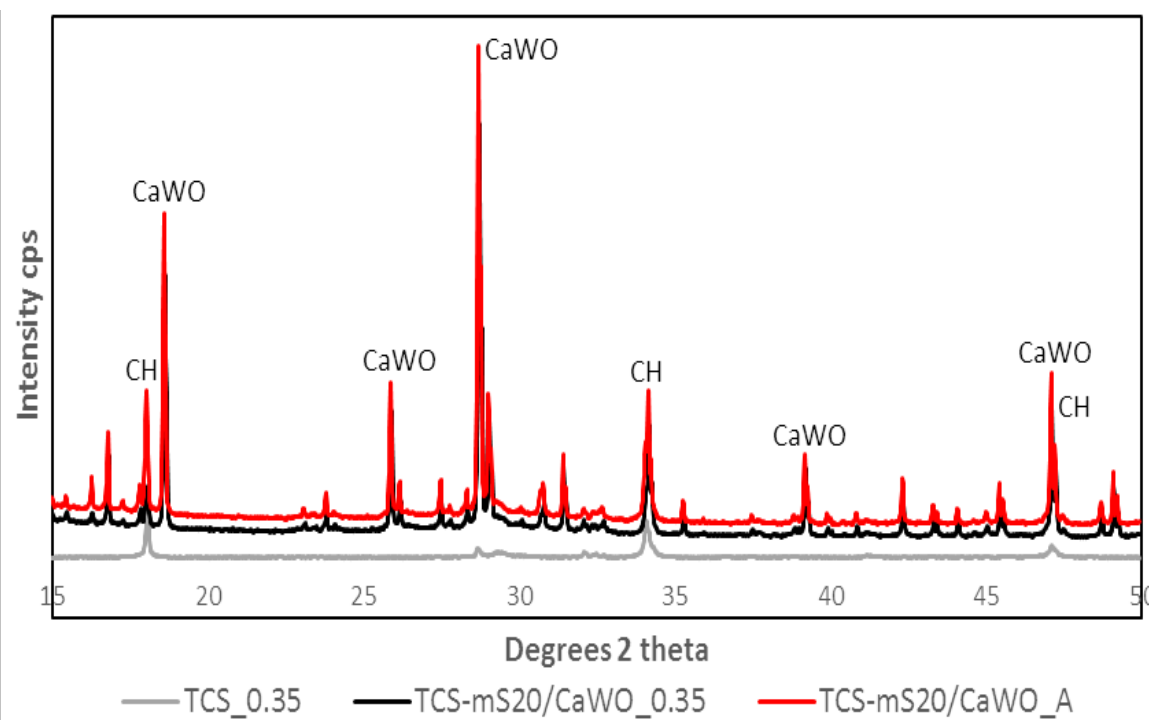

Figure 1d. X-ray diffraction plots of tricalcium silicate cement and test prototype materials with different radio-opacifiers and also incorporating 20\%microsilica mixed in a 0.35 water: powder ratio or an adjusted ratio after immersion in Hank's balanced salt solution for 1 week. $\mathrm{CH}$ : calcium hydroxide. 


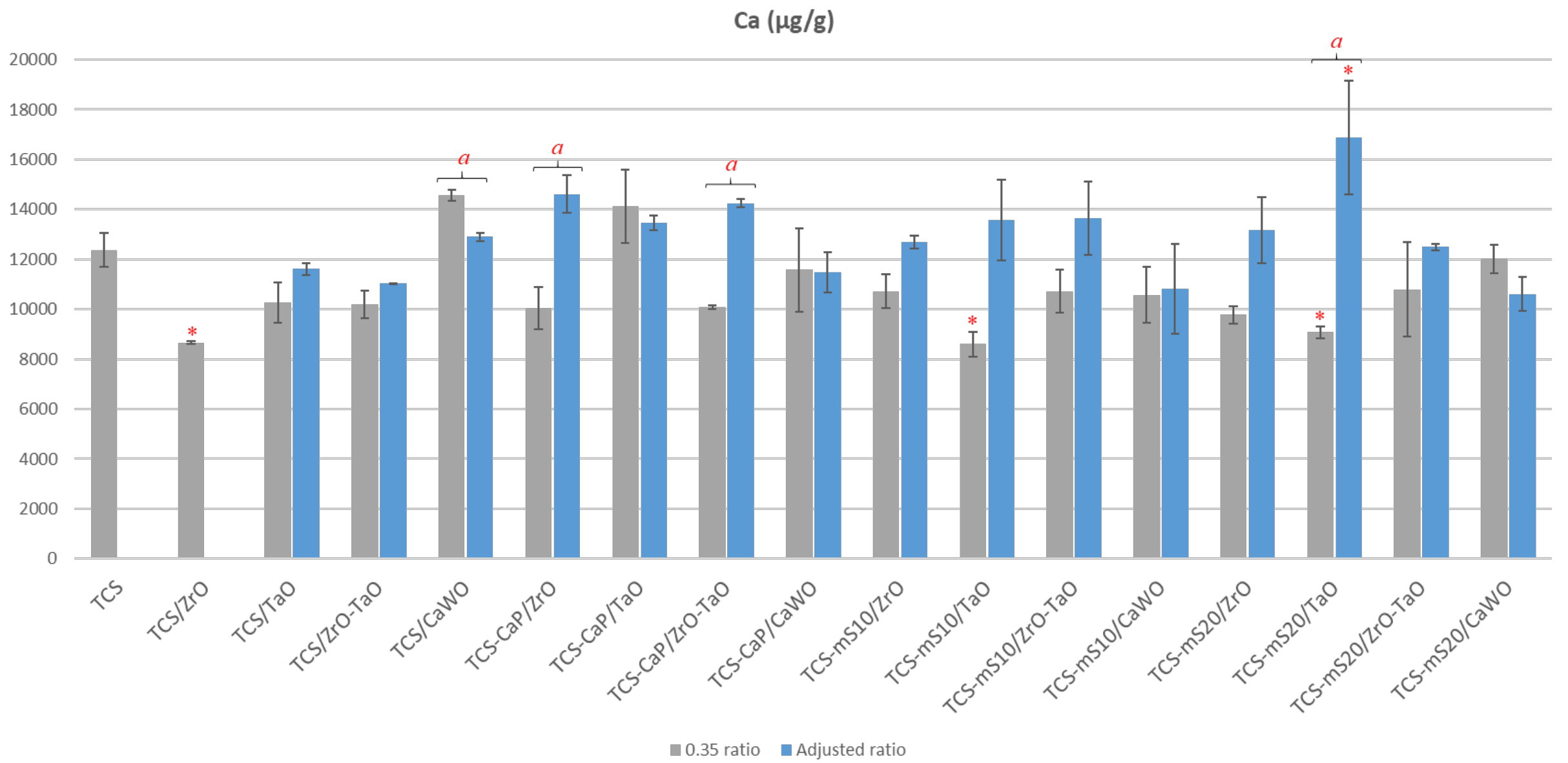

Figure 2. Calcium release of prototype materials mixed in a 0.35 water: powder ratio or an adjusted ratio after immersion in Hank's balanced salt solution for 1 week. Asterisk indicates significant difference with the TCS_0.35 ( $p<0.05)$; the latin letter ' $a$ ' represents statistical difference between materials with the same components and different water: powder ratios $(p<0.05)$. 

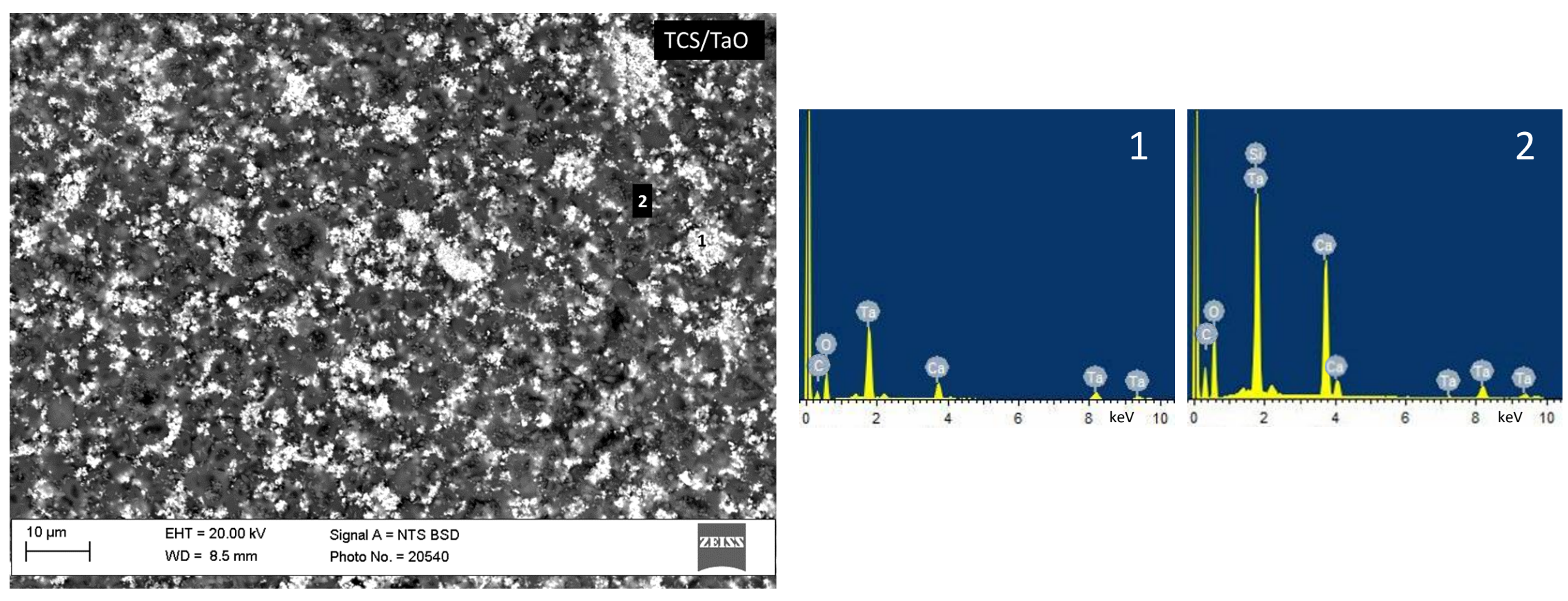

a)
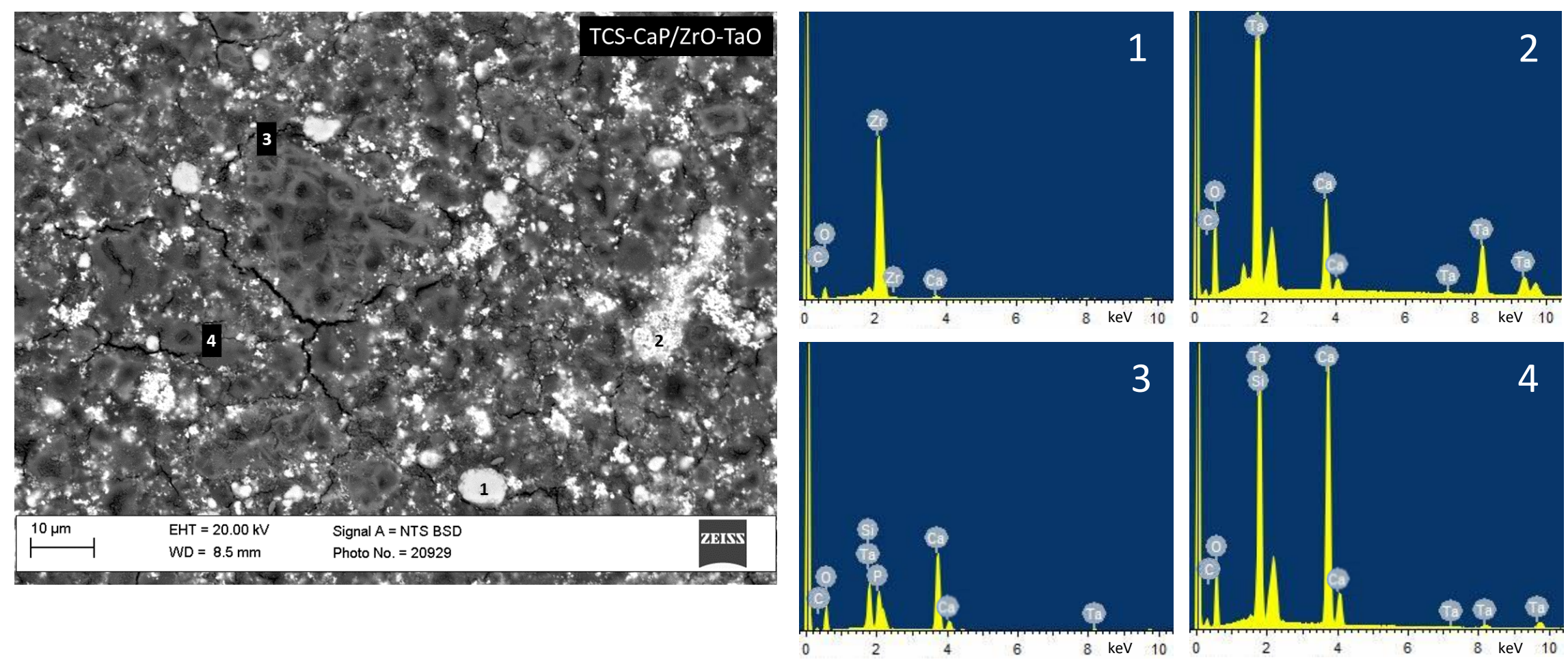

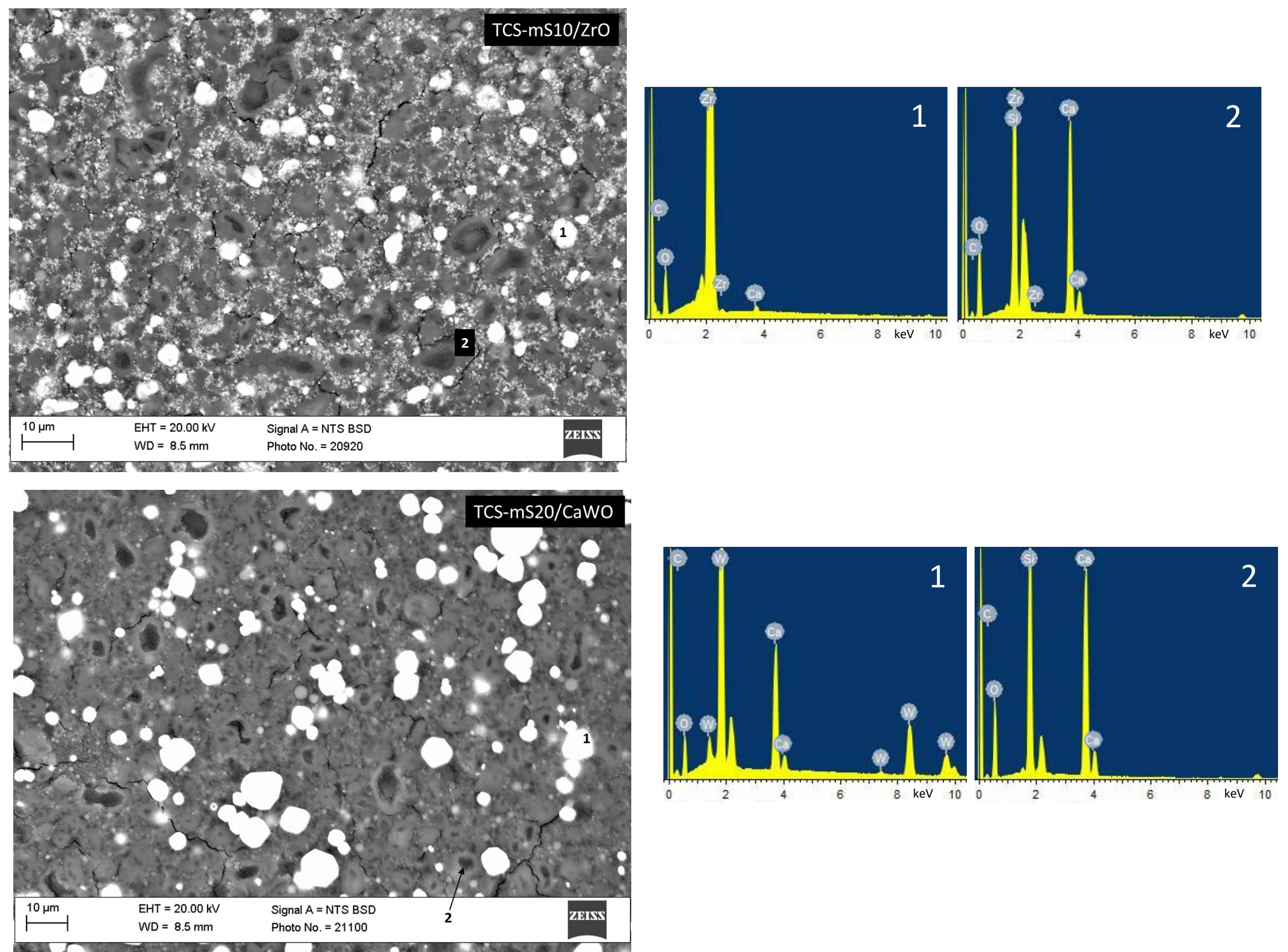

d)

Figure 3. Representative back-scatter scanning electron micrographs of polished sections of prototypes with different additives and radioopacifiers (2500X magnification) showing microstructural components and energy-dispersive spectroscopic scans of selected spectrums (a-d). 